\title{
Roles of multiple accessory molecules in T-cell activation
}

\author{
Gijs A. van Seventer, Yoji Shimizu and Stephen Shaw
}

\author{
National Institutes of Health, Bethesda, Maryland and University of Michigan Medical School, \\ Ann Arbor, Michigan, USA
}

\begin{abstract}
Accessory molecules expressed on T cells can mediate adhesion between $T$ cells and other cells, or the extracellular matrix. The same T-cell accessory molecules participate in a dialogue with their ligands (counter-receptors) on the antigen-presenting cells, and elicit signals that determine the specifics of activation and subsequent differentiation of the $T$ cells and antigen-presenting cells.
\end{abstract}

Current Opinion in Immunology 1991, 3:294-303

\section{Introduction}

The T-cell receptor (TCR)-CD3 complex is critical to $T$-cell recognition because it confers antigen-specificity and initiates a cascade of biochemical events that are essential to T-cell activation (see [1] for a review). Binding and aggregation of TCR-CD3 by MHC and antigenic peptide or by $\mathrm{CD} 3$ monoclonal antibody (mAb) is, in most model systems, insufficient to activate resting $T$ cells, and may even induce tolerance (see [2] for a review). Multiple $\mathrm{T}$-cell surface molecules have been demonstrated to be capable of co-stimulation that is sufficient to fully ac tivate $\mathrm{T}$ cells, in combination with a TCR-CD3-mediated signal. Typically, this co-stimulation is demonstrated using experimental systems in which the presence of mabs specific for these molecules facilitates activation of $T$ cells by anti-TCR-CD3 mAbs or by specific antigen.

Since Clark reviewed data on four such molecules in 1986 [3], the number of co-stimulatory or accessory molecules has mushroomed to more than 20 (for a review see [4]). The excitement regarding mAb-mediated co-stimulation results from the presumption (which we share) that the mabs often mimic the natural ligand (defined here as the molecule expressed on an apposing cell that interacts with the $\mathrm{T}$-cell accessory molecule). The next step is obligatory, and is the definition of the relevant ligands and analysis of their capacity to mediate co-stimulation (the best defined are summarized in Fig. 1). Ligand characterization has been achieved for three receptor-ligand pathways that participate in T-cell antigen-presenting cell (APC) interaction: CD2-lymphocyte function-associated antigen (LFA)-3, LFA-1-intercellular adhesion molecule (ICAM)-1 and CD28-B7. We will review the progress that has been made in understanding these pathways. Comment is also made on parallels between these pathways and recently-defined adhesion pathways that mediate
T-cell interaction with extracellular matrix (ECM): very late antigen (VLA)-4-fibronectin (FN), VLA-5-FN, VLA6-laminin (LN), VLA-3-collagen, CD26-collagen) (see Fig. 1).

Superficially, there appears to be a great redundancy of molecules involved in co-stimulation. On reflection, however, it becomes apparent that $\mathrm{T}$-cell interactions with APCs are complex multistep processes that critically depend on both adhesion and signal transduction. As an illustration of this complex dialogue, we will also review in detail, the interaction of T cells with two kinds of APC, monocytes and B cells, with a particular emphasis on the roles of the T-cell molecules CD2, LFA-1 and CD28. In each case, we find that multiple signals are integrated by the participating cells and are used to program the differentiation events that result from the T-cell-APC encounter.

\section{Interplay between adhesion and activation}

\section{Many adhesion molecules are involved in T-cell co-stimulation}

Adhesion molecules are ideally situated to transduce in formation to the cell during the adhesion event i.e. to in form the cell thai contact has been made and to convey information regarding the character of the apposing cell. This realization, obvious only in retrospect, emerges from the finding that many adhesion molecules also mediate co-stimulation. Thus adhesion molecules are not simply 'glue', but rather transducers of information.

$\mathrm{CD} 2$ was the first T-cell molecule for which dual roles in adhesion and activation were clearly established (for review see [5]). Recent studies have refined these concepts. Because of the dramatic capacity of $\mathrm{CD} 2$ to ac-

\footnotetext{
Abbreviations

APC - antigen-presenting cell; $\left[\mathrm{Ca}^{2+}\right]_{i}-$ intracellular concentration of $\mathrm{Ca}^{2+} ; \mathbf{E C M}$-extracellular matrix; EAM - endothelial leukocyte adhesion molecule; FN-fibronectin; ICAM-intercellular adhesion molecule; IL-interleukin; LFA-lymphocyte function-associated antigen; $\mathbf{L N}$-laminin; Th-T helper; mAb-monoclonal antibody; PKC-protein kinase C; TCR-T-cell receptor; TNF-tumor necrosis factor; VLA-very late antigen.
} 


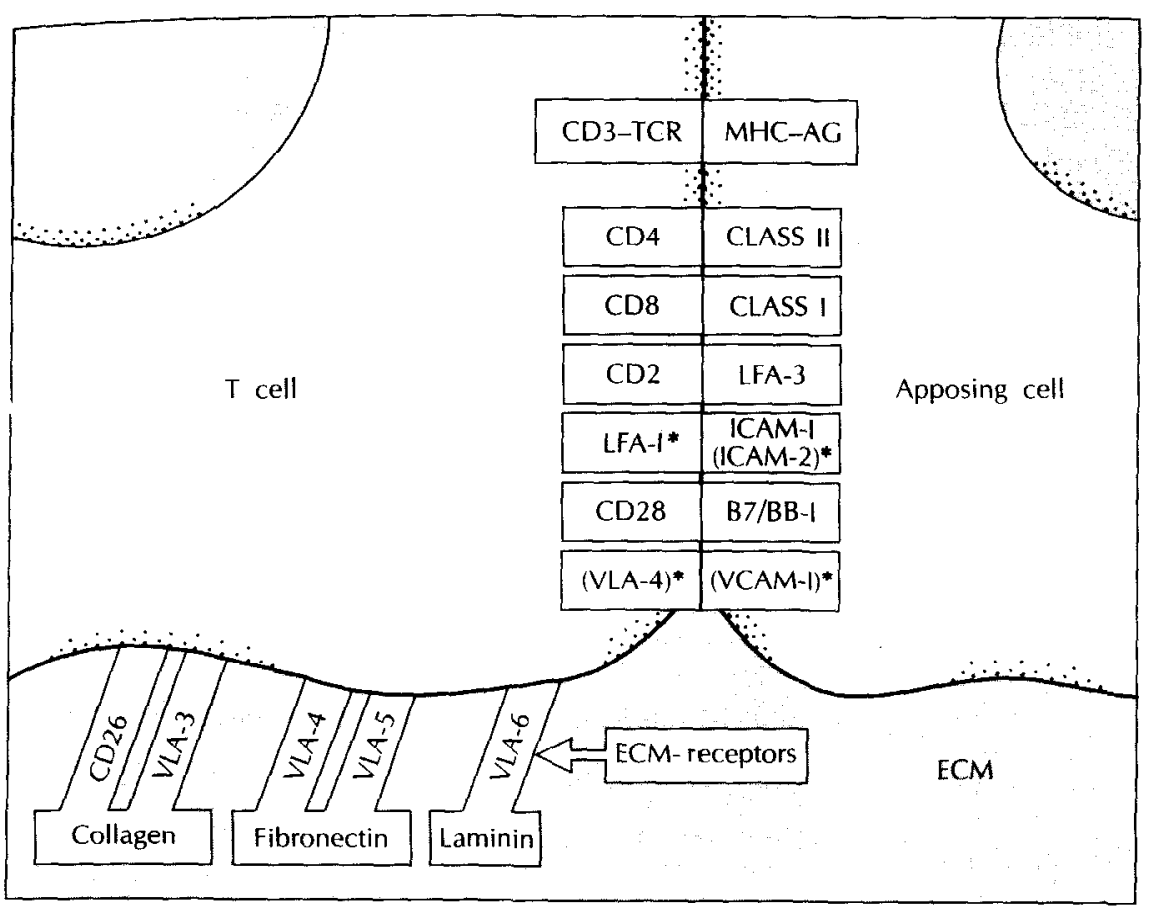

Fig. 1. Accessory molecules implicated in adhesion and co-stimulation of resting T-cells. *LFA-1-1CAM-2 and VLA4/VCAM-1 have only been shown to play a role in adhesion. $\mathrm{Ag}$, antigen; ECM, extracellular matrix; ICAM, intercellular adhesion molecule; LFA, lymphocyte function-associated antigen; $\mathrm{MHC}$, major histocompatibility complex; VLA, very late antigen. tivate cells, the importance of its adhesive role is of ten under emphasized. However, recent studies suggest that CD2-IFA-3 adhesion mediates substantial augmentation of antigen-specific $T$-cell responses [6•]; specifically, augmentation is observed following transfection of $T$ cells with recombinant $\mathrm{CD} 2 \mathrm{cDNA}$ lacking the cytoplasmic domain responsible for the signalling capacity of the molecule. There is controversy over whether the func. tional synergism between CD2 and CD3 is partly a result of a physical association between the molecules: whereas one study provided evidence of physical association between $\mathrm{CD} 2$ and $\mathrm{CD} 3$ by co-precipitation analysis [7•], another failed to see co-clustering of CD3 with CD2 in the area of cell-cell interaction, during the antigen-independent phase of conjugation [8*].

In contrast to CD2, LFA-1 historically has been recog nized principally as an adhesion molecule (for review see [9]). LFA-1 mediates binding not only to activationinducible ligand ICAM-1 but also to an additional ligand ICAM-2 [10 $]$, which is constitutively expressed on endothelial cells. Recent studies with ICAM-1 transfectants $\left[11^{\bullet}, 1^{\bullet}\right]$ or with purified ICAM- $1\left[13^{\bullet}, 14^{\bullet}\right]$ indicate that the LFA-1-ICAM-1 pathway is also involved in co-stimulation of TCR-CD3-mediated T-cell activation. Although the mechanism of co-stimulation remains unclear, evidence for signalling through LFA-1 comes from studies in which crosslinking of T cell LFA-1 with mAbs was found to induce increased intracellular $\mathrm{Ca}^{2}+\left[\mathrm{Ca}^{2+}\right]_{\mathrm{i}}$ concentrations $\left[15^{\bullet}, 16^{\bullet}\right]$.

The CD28 molecule was initially identified as a potent costimulator of T-cell activation (for a review see [17]). Re- cently, the CD28 ligand was identified as the previously defined B-cell activation antigen B7 (or BB-1) [18••]. A dual role for the CD28-B7 pathway in adhesion and costimulation has been demonstrated by the demonstration that immobilized purified $B 7$ protein mediates $T$-cell adhesion and co-stimulation that results in T-cell proliferation and cytokine production [19॰]. Studies of costimulation by mAb-ligation of CD28 have identified several mechanisms by which CD28 influence T-cell activation. Firstly, aggregation of $\mathrm{CD} 28$ appears to activate a phospholipase $C$ that is functionally different from that activated by TCR-CD3 [20*]. Secondly, there is a unique transcriptional regulatory sequence in the interleukin (IL)-2 gene that is involved in CD28-induced activation [21.], and thirdly, CD28 co-stimulation partly acts by stabilization of cytokine mRNA (e.g. IL-2) [22•].

The concept of co-stimulation has been expanded by studies of ligands that are neither cytokines nor cell-surface molecules, but are ECM proteins. Co-stimulation by five such receptor-ECM interactions has been described. Four of the $\mathrm{T}$-cell adhesion receptors for these ligands are members of the VLA or $\beta 1$ (CD29) subfamily of integrins (for a review see [23]). The details are complex because some of these receptors have more than one ligand, and one ligand may have various receptors. For example, T-cell VLA-4 and VLA-5 bind to distinct sites on the ECM protein, FN $\left[24^{\circ}, 25^{\circ}\right]$. The two other Tcell integrin-mediated interactions implicated in co-stimulation are VLA-3 binding to collagen [26*] and VLA-6 binding to $\mathrm{LN}\left[25^{\circ}\right]$. Collagen also mediates co-stimulation via the CD26 molecule (or Tp103, DPPIV) [26॰], 
which is not a member of the integrin family but is a dipeptidylpeptidase. All these receptor-ligand combinations co-stimulate in $\mathrm{CD} 3 \mathrm{mAb}$-mediated T-cell activation $\left[26 \cdot-30^{\bullet}\right]$.

The biological relevance of co-stimulation by ECM molecules remains to be defined. One interesting possibility is that the ECM environment that surrounds a $\mathrm{T}$ cell migrating through tissue, influences its ability to be activated, or its subsequent differentiation (see below). The strong parallels between T-cell interaction with ECM and with other cclls is emphasized by the finding that VLA-4 is not only an FN receptor but also binds the cell-surface molecule VC.AM-1 [31•]. The mode by which these ECM receptors co-stimulate is largely unknown although it has recently been shown that VLA-5-mediated co-stimulation by FN induces the IL-2 gene transcription factor AP-1 [32•].

\section{Adhesion is acutely regulated by activation}

T-cell adhesion is closely interconnected with T-cell activation. Not only does adhesion influcnce T-cell stimulation as mentioned above, but the activation state of the $T$ cell also affects adhesion. The first detailed de. scription of this active interplay between TCR-CD3-mediated activation and adhesion was given by Dustin and Springer [33**] for LFA-1 binding to ICAM-1. Subsequently TCR-CD3-mediated activation was also found to affect binding via the related integrins VLA-4, VLA-5 and VLA-6 [25*], and a fifth molecule with a different structure, the CD8 glycoprotein [34*]. This contrasts with CD2-mediated adhesion, which appears to be independent of acute activation [35॰]. For each of the four integrins, TCR-CD3 crosslinking results in a rapid $(<10 \mathrm{~min}$ ) increase in avidity of adhesion without an increase in cell-surface expression of the particular receptors; furthermore, the augmentation of adhesion is transient. Although these results were obtained in artificial systems, they make a great deal of sense in their prediction that antigen-specific $T$-cell recognition results in augmented adhesion that stabilizes the T-cell interactions with apposing cells (or ECM) for a period of time. The molecular mechanism(s) that accounts for these changes is not understood and may be regulated in part, by signals from the T-cell receptor via protein kinase $C$ (PKC) because they are inhibited by the PKC inhibitor staurosporine ([33**], and Y Shimizu unpublished data). Among many possibilities, two are particularly attractive explanations for the regulation of avidity. One is that in. tracellular events affecting the integrin molecule (such as phosphorylation) induce conformational changes of the ligand-binding domain that alter its affinity for ligand. The other is that intracellular events result in micro-clustering (either by self association or by cytoskeletal linkage), which augments local avidity [36].

Activation via CD2 alone also appears to affect integrinmediated adhesion. Stimulating pairs of CD2 mAbs results in a rapid, increased avidity of LFA-1 and VLA in- tegrins for their respective ligands $\left[25 \bullet, 37^{\bullet}\right]$. Although the induced binding of T cells by TCR-CD3 $\left[33^{*}, 38\right]$ is quite transient ( $\approx 30-40 \mathrm{~min}$ ), adhesion mediated via $\mathrm{CD} 2$ stimulation is longer in duration ([37•], and $\mathrm{Y}$ Shimizu, unpublished data).

T-cell adhesion to APCs constitutes a bilateral process because generally, APCs also express adhesion molecules. Thus, it is not surprising to find that for the APC, like the $T$ cell, activation events may alter the avidity of adhesion molecules. For example, analogous to TCR-CD3-mediated stimulation of $\mathrm{T}$ cells, crosslinking of HLA class II antigens on monocytes induces high avidity LFA-1 function $\left[39^{\circ}\right]$.

\section{Adhesion is regulated by differentiation}

Adhesion is influenced not only by immediate stimulation of the $\mathrm{T}$ cell, but also by the differentiation state of the $T$ cell and the apposing cell. Human memory $T$ cells express significantly higher levels of an array of adhesion receptors and ligands, including LFA-1, ICAM-1, CD2, LFA-3 [40], CD26 [41•] VLA-4, VLA-5 and VLA-6 [25•] than do naive cells. Such regulation of expression by T-cell differentiation suggests that their function is critical to the differentiated function of those cells. In vitro studies [38] show that upon acute activation via TCR-CD3 or CD2memory cells bind more than naive cells to purified ligands. In vivo, Mackay et al. [42*] have demonstrated that naive cells recirculate preferentially through lymph nodes whereas memory cells recirculate primarily through nonlymphoid tissue. This suggests that homing and migration patterns of $\mathrm{T}$ cells depend on the differentiation state of the cell.

The ligands for T-cell adhesion molecules are also dramatically regulated. Activation results in induction of B7 on B cells and ICAM-1 on monocytes, which is critical to their interaction with $T$ cells (see below). The most complete and elegant studies of regulated expression of ligands have been carried out on endothelial cells. $T$ cells bind to endothelium via molecular pathways that include: LFA-1-ICAM-1; LFA-1-ICAM-2; and VLA-4-VCAM-1. $A$ fourth adhesion pathway exists for a memory subset of $\mathrm{CD}_{4}^{+} \mathrm{T}$ cells and is mediated by the activation-induced ligand, endothelial leukocyte adhesion molecule (ELAM)-1 on endothelial cells [43-45*]. The receptor on T cells, however, has not yet been defined. ICAM-2 is expressed on resting endothelium and its transcription is not augmented with inflammatory cytokines $\left[10^{\bullet}, 46\right]$. In contrast, resting endothelium cells express ICAM-1 minimally, and do not express VCAM-1 or ELAM-1 at all $\left[43^{\circ}, 46,47^{\circ}\right]$. All three molecules are rapidly induced by IL-1 $\beta$ or tumor necrosis factor (TNF). Interferon, how. ever, selectively induces ICAM-1, and IL-4 only increases VCAM-1 ([48*], and W Newman, personal communication) and downregulates ICAM-1-expression [48 ${ }^{\circ}$. Thus, T-cell capture by endothelium, the critical first step in $\mathrm{T}$ cell migration into tissue, may be exquisitely regulated by the molecules expressed by endothelial cells. 


\section{A dialogue between $T$ cells and APCS}

An antigen-specific interaction of a $\mathrm{T}$ cell with an APC can be regarded as a highly specific sequence of events, which shows much resemblance to some of nature's complex 'mating rituals'. Specific interaction between a $T$ cell and an APC involves a bilateral exchange of signals, which triggers a biochemical cascade of events that lead to specific activation and differentiation not only of the ' $\mathrm{T}$ cell, but also of the apposing APC. Similarly, T-Bcell interactions may lead to activation and differentiation of B cells. Incomplete exchange of information may lead to a failure of complete activation, and may even induce the $T$ cell to become anergic/tolerized to the improperly presented antigen.

\section{$T$ cell-monocyte interaction}

Many antigen-specific T-cell activation models use monocytes as APCs. Figure 2 collects information from various systems into a proposed sequence of signal exchanges that ultimately lead to T-cell proliferation and monocyte cytokine secretion by the monocyte. Initial, weak cell contact (Fig 2a) occurs between a $T$ cell and monocyte; this initial contact may be mediated by LFA-1 (which has a low but detectable affinity for ICAM-1 on resting T cells) or by other receptor-ligand interactions. TCR-CD3 engagement by $\mathrm{MHC}$-antigen (Fig. $2 \mathrm{~b}$ ) results in signal delivery in both the $T$ cell and the monocyte. In the $T$ cell, TCR-CD3 stimulation results in the induction of high avidity LFA-1 [33**], which considerably strengthens the $\mathrm{T}$ cell-monocyte interaction. Similarly, crosslinking of $\mathrm{MHC}$ antigen on the monocyte induces high avidity LFA-1 on the monocyte [39०]. This strengthened IFA1 -dependent interaction may allow weaker adhesive interactions, such as CD2-LFA-3, to occur.

The ability of purified CD2 to induce IL 1 secretion from monocytes [490.] suggests that such interactions may be critically important in the induction of IL- 1 mRNA. Additional or alternative mechanisms for initial IL-1 mRNA production may also exist, as Landis et al. [50*] have reported that a cell contact-mediated signal provided by CD3 mAb-stimulated $T$ cells (but not resting $T$ cells) is important for the initial phase of IL-1 mRNA induction. This cell contact-mediated signal does not require new protein synthesis [50*]. Further activation of the mono cyte leads to important cell-surface changes on the monocyte and ultimately to cytokine secretion (Fig. 2c). Such activation may be mediated by cell contact-mediated signals of the $T$ cell, and by soluble factors produced by the activated $T$ cell [50.] or other cells in the microenvironment.

Kawakami et al. [51••] have demonstrated in a monocytedependent model system that optimal T-cell activation requires crosslinking of the TCR-CD3 complex, the presence of the cytokines IL-1 $\beta$ and IL-6, and and IFN- $\gamma$-induced membrane-bound co-stimulatory signal expressed on monocytes. Studies of the molecules involved in this co-stimulation demonstrate a requirement for both the LFA-1-ICAM-1 and CD2-LFA-3 pathways, and increased ICAM-1 expression on the activated monocytes (GA van Seventer et al. unpublished data). This model system described by Kawakami et al. [51॰0] shows much resemblance to the in vitro models for induction of clonal anergy in murine $\mathrm{T}$ helper (Th)1-type clones. In both, a cell contact-mediated signal, which cannot be replaced by the addition of various cytokines is required in addition to MHC and antigenic peptide, to elicit optimal T-cell stimulation, (see [2] for review). The exact nature of the membrane-bound co-stimulatory signals in both systems is not well resolved. In our proposed model (Fig. 2) the expression of the membrane-bound co-stimulatory signal by the monocyte, along with induced secretion of $c y$. tokines such as IL-1 $\beta$ and IL- 6 by the activated monocyte, finally leads to IL-2-dependent proliferation and differentiation of the $\mathrm{T}$ cell (Fig. $2 \mathrm{~d}$ ).

\section{T-cell-B-cell interaction}

A similar dialogue occurs in T-B-cell interaction, although some of the molecules involved are different (see Fig. 3). Even though we have attempted to simplify the sequence of events for clarity, the $\mathrm{T}-\mathrm{B}$-cell interaction currently involves at least 14 different surface molecules on the two apposing cells! As with monocytes, weak adhesion (Fig. 3a) is followed by antigen-specific interaction of TCR-CD3 with antigen-MHC, and the delivery of signals to both the $T$ cell and $B$ cell (Fig. 3b). Direct evidence for a role of the CD2-LFA-3 pathway (Fig 3b) is not yet established. Although not directly demonstrated, triggering of the antigen-MHC complex on the B cell may increase the adhesion of the $B$ cell to the $T$ cell; in addition, recent studies indicate that crosslinking of class II antigens on B cells results in the induced expression of the CD28 ligand B7/BB-1 [52•], which is likely to be critical in the later steps of this interaction (see below).

On the T cell, TCR-CD3 activation results in high avidity LFA-1 function and increased T-cell adhesiveness [33*•]. Studies of B-cell activation also suggest that a signal provided by $\mathrm{CD} 3 \mathrm{mAb}$-activated $\mathrm{T}$ cells (but not resting $\mathrm{T}$ cells) is necessary for B-cell entry into the G1 phase of the cell cycle. This signal apparently requires synthesis of a new protein (Protein X in Fig. 3) $\left[53^{\bullet \bullet, 54 \bullet, 55 \bullet, 56 \bullet] . ~}\right.$ Thus, (Fig. 3c), denotes the delivery of a signal to the partially activated B cell by Protein X. Step (Fig 3d) involves B-cell co-stimulation of the $T$ cell and is proposed as a result of evidence that activated, but not resting $B$ cells are effective accessory cells for T-cell activation [57]. Recent studies in murine models of in vitro induction of clonal anergy suggest that resting B cells lack the capability of highly enriched, activated B cells to provide the membrane-bound co-stimulatory signal necessary to prevent induction of clonal anergy [58*]. The nature of any membrane-bound co-stimulatory signal(s) on activated B cells remains largely undefined. Recently however, two studies have demonstrated that mAbs specific for $\mathrm{CD} 28$ or $\mathrm{B} 7$ inhibit allo-activation of $\mathrm{CD}^{+}$ T cells by B hymphoblasts $\left[52 \bullet, 59^{\bullet}\right]$, which makes B7/BB1 a plausible candidate for such a membrane-bound co- 


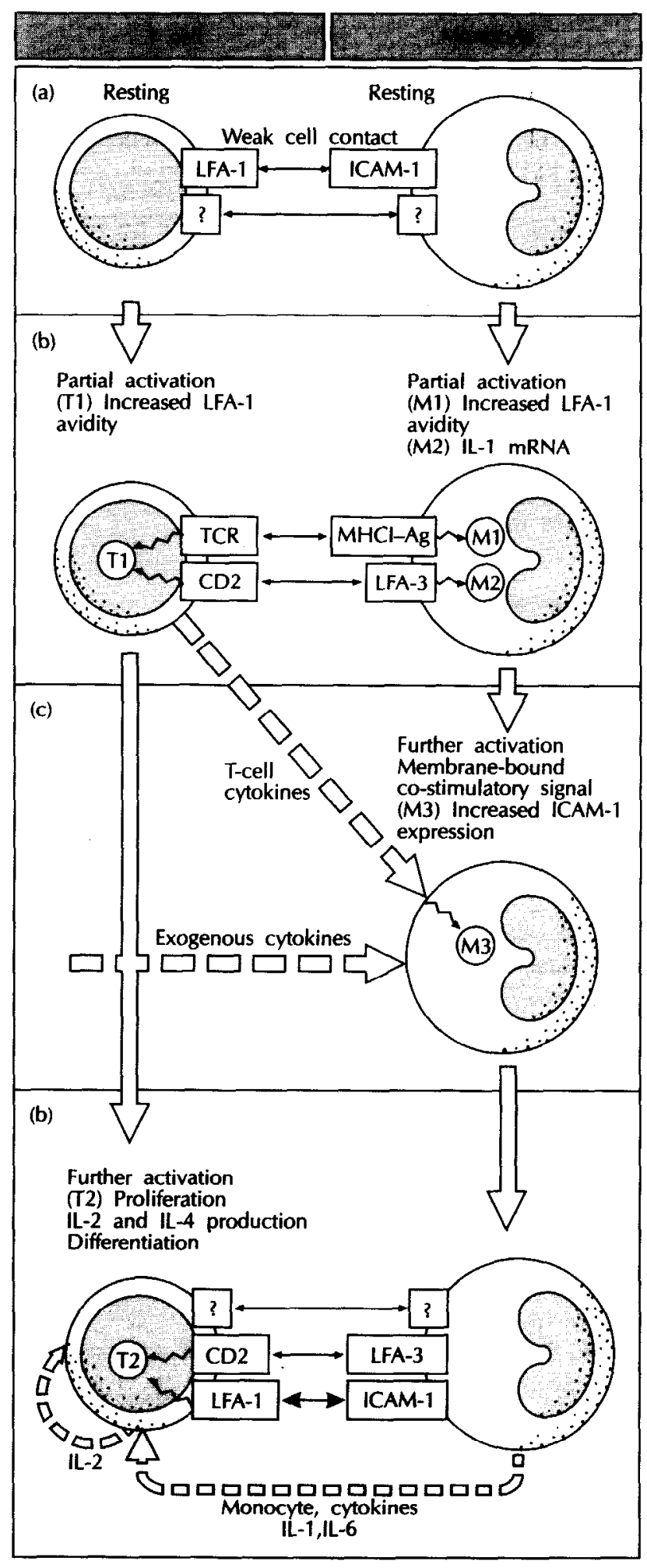

Fig. 2. T cell-monocyte bilateral interaction. Schematic depiction of the multiple pathways involved in T-cell-monocyte interaction (see accompanying text on T-cell-monocyte interaction for further details). Ag, antigen; ICAM, intercellular adhesion molecule; IL, interleukin; LFA, lymphocyte function-associated antigen; MHC, major histocompatibility complex; TCR, T-cell receptor. stimulatory molecule on B cells. Not surprisingly, the LFA1-ICAM-1 [60.] and CD2-LFA-3 [61] pathways have also been implicated in this co-stimulation, and additional cytokines produced by activated B cells may further facilitate activation of the $\mathrm{T}$ cell.

Finally, B-cell co-stimulation of the $\mathrm{T}$ cell results in proliferation and differentiation of the $\mathrm{T}$ cell. Initially $\mathrm{T}$-cell secretion of IL- 4 drives the $B$ cell into the $S$ phase of the cell cycle (Fig. $3 e$ ) $\left[53^{* \bullet}, 54^{\bullet \bullet}, 55^{\circ}, 56^{\bullet}\right]$. Subsequently, the $T$ cell produces IL-5, which induces IgM production and further isotype switching (Fig 3f) $\left[55^{\bullet}, 56^{\bullet}\right]$. T-cell-induced differentiation of $\mathrm{B}$ cells is also shown to be dependent on the LFA-1-ICAM-1 [60'], CD2-LFA-3 [61] and CD28-B7 [59०] pathways. It is unclear, however, whether this solely reflects a role for these pathways in co-stimulation of $\mathrm{T}$ cells, leading to production of cytokines such as IL- 4 and IL- 5 by the T cell, or a more direct involvement of these pathways with differentiation of B cells. Thus this complex bi-directional interplay leads to proliferation and differentiation of both interacting cells.

\section{Integration of multiple signals influences differentiation and effector function of $T$ cells}

It is an appealing concept that the multiplicity of molecular interactions between T cell and APC determine the specifics of activation, and subsequent differentiation of the T cell and APC. Support for this concept comes from evidence that various co-combinations of stimuli generated by different accessory molecules induce different responses from $\mathrm{T}$ cells and different $\mathrm{T}$-cell subsets respond differently to stimulation via accessory molecules. The former is of particular relevance to the present discussion. Such regulation of alternative T-cell effector functions by co-stimulatory molecules is illustrated by a recent study that describes $I L-1 \alpha$ production by $T$ cells. Late ( $>48 \mathrm{~h}$ ) IL-1 $\alpha$ secretion is achieved by stimulation through combinations of $\mathrm{CD} 2$ and $\mathrm{CD} 28$ mAbs (or CD3 and $\mathrm{CD} 28 \mathrm{mAbs}$ ), but not by any of these mAbs in isolation, although they are able to induce $\mathrm{T}$-cell proliferation and TNF- $\alpha$ secretion [62•]. One can also envision that co-stimulatory molecules may regulate different effector functions. For example, Eljaafari et al. [63'] demonstrated that two distinct pairs of $\mathrm{CD} 2 \mathrm{mAbs}$ (which possibly mimick different ligands), each of which induce different second messengers, stimulated bifunctional $\mathrm{CD}^{+}{ }^{+}$ T-cell clones to perform either helper or cytolytic func tions. These data support the hypothesis that the combination of accessory-cell ligands expressed by an apposing APC will strongly shape the subsequent T-cell differentiation: ECM ligands in the vicinity may also contribute. 


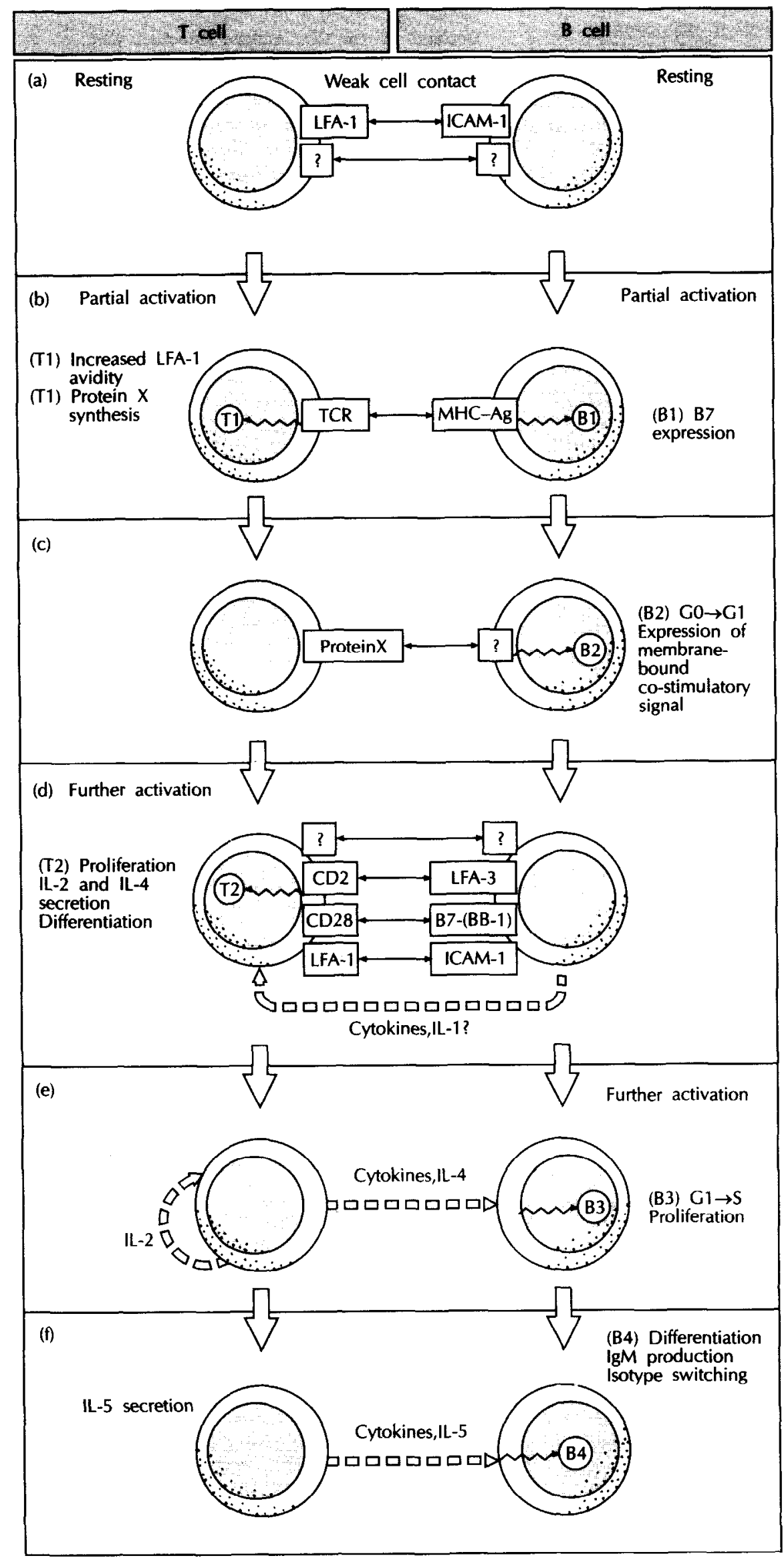

Fig. 3. $T$ cell $-B$ cell bilateral interaction. Schematic depiction of the multiple pathways involved in $T$ cell-B cell interaction (see accompanying text on $T$ cell-B cell interaction for further details). $\mathrm{Ag}$, antigen; ICAM, intercellular adhesion molecule; LFA, lymphocyte function-associated antigen; IL, interleukin; MHC, major histocompatibility complex; TCR, T-cell receptor. 


\section{Conclusion}

Given the interest and progress in accessory molecules, this review has barely scratched the surface. It has dealt with selected studies that illustrate the following themes, which we view as important in a general understanding of accessory molecules in T-cell activation. Firstly, there is a multiplicity of molecules involved in T-cell activation. Secondly, there is a dynamic interplay between adhesion and activation wherein adhesion molecules mediate signal transduction and intracellular signals modulate the character of the adhesion. Thirdly, cell-cell interactions such as T cell with APC involve a multistep dialogue between receptors on the $T$ cell and their ligands on the APC. Finally multiple signals from these diverse molecular interactions are integrated by poorly defined molecular processes within the cells, which then determine the specifics of activation and subsequent differentiation of the $\mathrm{T}$ cell and APC.

\section{Acknowledgements}

We are thankful to Drs AA Brian, EA Clark, NK Damle, B Dupont MK Jenkins, L. Koulova, P Kuhlman, PS Linsley, JA Ledbetter, P Moingeon, C Morimoto, W Newman, RJ Noelle, and EL Reinherz for sharing with us unpublished data and papers prior to publication. The critical reading of the manuscript by Drs RE Gress and RJ Hodes is highly appreciated. GA van Seventer is a visiting associate supported by the Fogarty Exchange Program. Y Shimizu was supported by Damon-Runyon Walter Winchell Cancer Research Fund Postdoctoral Fellowship DRG-993.

\section{References and recommended reading}

Papers of special interest, published within the anmual period of review, have been highlighted as:

- of interest

-. of outstanding interest

1. Clevers $H$, alarcon B, Wheman T, Terhorst C: The T Cell Receptor/CD3 Complex: A Dynamic Protein Ensemble. Annu Rev Immunol 1988, 6:629-662.

2. SCHWARTZ RH: A Cell Culture Model for $T$ Lymphocyte Clonal Anergy. Science 1990, 248:1349-1355.

3. CLARK EA, LEDBETTER JA: Amplification of the Immune Response by Agonistic Antibodies. Immunol Today 1986, $7: 267-270$

4. GEPPERT TD, DAVIS LS, GUR H, WACHOLTZ MC, LIPSKY PE: ACcessory Cell Signals Involved in T-cell Activation. Immuno Rev 1990, 117:1-66.

5. SHaw S, SHimiz Y: Two Molecular Pathways of Human T Cell Adhesion: Establishment of Receptor-ligand Relationship. Curr Opin Immunol 1988, 1:92-97.

6. MoIngeon P, Chang H, Waluner BP, StebBins C, Frey AZ, - REINHER EL: CD2-mediated Adhesion Facilitates T Lymphocyte Recognition Function. Nature 1989, 339:312-314.

Documents that by transfection of $\mathrm{CD} 2$ cDNA constructs lacking the cytoplasmic signal transduction element, adhesion of CD2 with LFA-3 alone can account for its facilitating effect on antigen-specific $T$-cell re sponses.
7. Brown MH, Cantrell DA, Brattsand G, Crumpton MJ, - Guluberg M: The CD2 Antigen Associates with the T-cell Antigen Receptor CD3 Antigen Complex on the Surface of Human T Lymphocytes. Nature 1989, 339:551-553.

Reports that $40 \%$ of the cell-surface $\mathrm{CD} 2$ molecules can be specifically co-pricipitated in association with the TCR-CD3 complex, when using a digitonin-based solubilization method.

8. Koyasu S, Lawton T, Novick D, Recny MA, Siliciano RF, - WaLNer BP, ReINHERz EL: Role of Interaction of CD2 Molecules with Lymphocyte Function-associated Antigen 3 in T-cell Recognition of Nominal Antigen. Proc Natl Acad Sci USA 1990, 87:2603-2607.

During conjugate formation, $\mathrm{CD} 2$ but not $\mathrm{CD} 3$ molecules are redistributed into the region of cell-cell interaction in an antigen-inde pendent way, suggesting that physical assuciation of $\mathrm{CD} 2$ with $\mathrm{CD} 3$ molecules is not essential for T-cell activation.

9. SPRINGER TA: Adhesion Receptors of the Immune System. Nature 1990, 346:425-434.

10. Staunton DE, DUSTTN ML, SPRINGer TA: Functional Cloning - of ICAM-2, a Cell Adhesion Ligand for LFA-1 Homologous to ICAM-1. Nature 1989, 339:61-64.

Functional cloning and identification of ICAM-2, a second ligand for LFA-1. Although this integral membrane-molecule has only two immunoglobulin-like domains they are closely related to the two most $\mathrm{N}$-terminal domains of the other LFA-1 ligand, ICAM-1.

11. Altmann DM, Hogg N, Trowsdale J, Wiukinson D: Cotrans- fection of ICAM-1 and HLA-DR Reconstitutes Human Antigen Presenting Cell Function in Mouse L Cells. Nature 1989, 338:512-514.

Reports that through transfection experiments, co-expression of ICAM-1 with HLA-DR on mouse fibroblasts is critical for effective HIA class-II-restricted and allo-specific human T-cell activadion.

12. SIU G, HedrICK SM, BRIAN AA: Isolation of the Murine Inter- cellular Adhesion Molecule 1 (ICAM-1) Gene. ICAM-1 enhances Antigen-specific T Cell Activation. I Immunol 1989, 143:3813-3820.

Isolation and characterization of murine ICAM-1 indicates that it has limited homology with human ICAM-1. Transfection of murine ICAM-1 augments APC function of MHC class-II fibroblasts.

13. Van Seventer Ga, Shimizu Y, Horgan KJ, Shaw S: The LFa-1 - Ligand ICAM-1 Provides an Important Costimulatory Signal for T Cell Receptor-mediated Activation of Resting $\mathrm{T}$ Cells. J Immunol 1990, 144:4579-4586.

Reports that affinity purified ICAM-1 provides co stimulation in a CD3 mAb-mediated proliferation model.

14. Kunlman P, MoY VT, Lollo BA, Brian AA: The Accessory

- Function of Murine ICAM-1 in T Lymphocyte Activation: Contributions of Adhesion and Co-activation. $J$ Immunol 1991, 146:1773-1782.

Murine ICAM-1 can function as a ligand for mLFA-1 in adhesion assays. Furthermore mICAM-1 enhances antigen-specific T-cell activation by affecting the dose-dependence of the response.

15. Pardi R, Bender JR, Dettori C, Giannazza E, Engleman

- EG: Heterogeneous Distribution and Transmembrane Signaling Properties of Lymphocyte Function-associated Antigen (LFA-1) in Human Lymphocyte Subsets. I Immunol $1989,143: 3157-3166$.

Documents signalling through LFA-1. LFA-1 crosslinking on peripheral $T$ cells results in phosphoinositide hydrolysis and a rise in $\left[\mathrm{Ca}^{2+}\right]_{\text {, }}$, when using CD11a (LFA-1 $\alpha$-chain) but not anti-CD18 (LFA-1 $\beta$-chain) mAbs.

16. WACHOLTZ MC, PATEL SS, LPSKY PE: Leukocyte Function- associated Antigen 1 is an Activation Molecule for Human T Cells. J Exp Med 1989, 170:431-448.

Neither CD11a nor CD18 mAbs alone, induced a rise in $\left[\mathrm{Ca}^{2+}\right]_{i}$ in T-cell clones, but combined with crosslinked CD3 mAbs a more prolonged calcium signal was observed than that observed when CD3 alone was crosslinked. The IL-2 production and DNA synthesis were 
also greatly enhanced under these conditions of crosslinking both $\mathrm{CD} 3$ and LFA-1.

17. JUNE CH, LEDBETTER JA, IINSLEY PS, THOMPSON CB: Role of the CD28 Receptor in T-cell Activation. Immunol Today $1990,11: 211-216$

18. LNSLEY PS, CLARK EA, LEDBETTER JA: T-cell Antigen CD28 Me 18. diates Adhesion with B Cells by Interacting with Activation Antigen B7/BB-1. Proc Natl Acad Sci USA 1990, 87:5031-5035. Cloning and identification of the B-cell activation antigen B7/BB-1 as a ligand for CD28. B7/BB-1 is an immunoglobulin-supergene family member, which when transfected into $\operatorname{COS}$ cells, mediates adhesion to CD28-transfected Chinese hamster ovary cells in a divalent cation-independent manner. This report provides the important and long-awaited identification of the natural ligand of CD28.

19. LNSLLY PS, BradY W, Grosmaire L, ARUFFo A, Damie NK - IEDBETtER J: Binding of the B Cell Activation Antigen B7 to CD28 Costimulates T Cell Proliferation and IL-2 mRNA Accumulation. J Exp Med 1991, 173:721-730.

Reports that $\mathrm{CD} 28$ binds to $\mathrm{B} 7 / \mathrm{BB}-1$ in the absence of other accessory molecules, and that interaction between CD28 and B7/BB-1 is co-stim ulatory for T-cell activation, resulting in proliferation and increased IL-2 transcription.

20. LEDBETTER JA, IMBODEN JB, SCHIEVEN GL, GrosmaiRe LS, - RABINOVtTCH PS, LINDSTEN T, ThOMPSON CB, JUNe CH: CD28 Ligation in T-cell Activation: Evidence for Two Signal Transduction Pathways. Blood 1990, 75:1531-1539.

Studies that exploit PMA-mediated inhibition indicate CD28 aggregation mediates its effect on inositol phosphate and stimulates $\left[\mathrm{Ca}^{2+}\right]$ rise through a phospholipase $\mathrm{C}$ activation pathway that is distinct from the TCR-CD3-linked pathway.

21. Fraser JD, Irving BA, Crabtret GR, Whiss A: Regulation of - Interleukin-2 Gene Enhancer Activity by the T Cell Accessory Molecules CD28. Science 1991, 251:313-316.

Stimulation of $\mathrm{CD} 28$ induces the formation of a protein complex that binds to a regulatory region of the IL-2 gene that is distinct from previously described regions.

22. Lindstein T, JUNE CH, LedBetter JA, STELIA G, THOMPSON

- $\quad$ CB: Regulation of Lymphokine Messenger RNA Stability by a Surface-mediated T Cell Activation Pathway. Science 1989, 244:339-343.

The CD28 activation pathway in T cells specifically regulates the stability of mRNAs for the lymphokines IL-2, INF. $\gamma$, TNF. $\alpha$ and GM.CSF.

23. SHIMLU Y, SHAw S: Lymphocyte Interactions with Extracellular Matrix. FASEB J 1991, in press.

24. Wayner ea, Garcla-Pardo a, humpiries MJ, mcDonald JA - CARTER WG: Identification and Characterization of the T Lymphocyte Adhesion Receptor for an Alternative Cell Attachment Domain (CS-1) in Flasma Fibronectin. / Cell Biol 1989, 109:1321-1330.

Identification of a new site of recognition for $\mathrm{T}$ cells, CS-1, that is on FN and is distinct from the Arg-Gly-Asp-peptide sequence.

25. Shimizu Y, van Seventer Ga, horgañ KJ, Shaw S: Regulated

- Expression and Function of Three VLA (beta-1) Integrin Receptors on T Cells. Nature 1990, 345:250-253.

Confirms and further investigates the concept of TCR-induced regulation of integrin function by demonstrating rapid induction of $T$-cell binding through VIA-4 and VLA-5 to fibronectin, and through VIA- 6 to LN, upon T-cell activation.

26. DANG NH, TORIMOTO Y, Schlossman SF, Morimoto C: Human

- CD4 Helper T Cell Activation: Functional Involvement of Two Distinct Collagen Receptors, IF7 and VLA Integrin Family. J Exp Med 1990, 172:649-652.

T-cell CD26 (DPP IV) and VLA-3 both bind to collagen and both provide co-stimulation in a $\mathrm{CD} 3 \mathrm{mAb}$-mediated proliferation system.

27. NOJMA Y, HuMPHRIES MJ, MOULD AP, KOMORNA A, YAMADA - KM, Schlossman SF, Morimoto C: VLA-4 Mediates CD3- dependent CD4 + T Cell Activation Via the CS1 Alternatively Spliced Domain of Fibronectin. J Exp Med 1990, 172:1185-1192

T cell VLA-4 provides co-stimulation by binding to the CS-1 region of FN in a CD3 mAb-mediated proliferation model.

28. MatsuYama T, Yamada A, KaY J, Yamada KM, AKIYAMa SK, - SChlossman SF, MORIMOTo C: Activation of CD4 Cells by Fibronectin and anti-CD3 Antibody. A Synergistic Effect Mediated by the VLA-5 Fibronectin Receptor Complex. J Exp Med 1989, 170:1133-1148.

T cell VLA-5 provides co-stimulation by binding to an Arg-Gly-Asp-peptide serulence $\mathrm{On} \mathrm{FN}$ in a CD3 mAh-mediated proliferation model.

29. Shimizu Y, van Seventer Ga, horgan KJ, Shaw S: Costimu. - lation of Proliferative Responses of Resting CD4 + T Cells by the Interaction of VLA-4 and VLA-5 with Fibronectin or VLA-6 with Laminin. I Immunol 1990, 145:59-67.

Resting $\mathrm{CDi}^{+}{ }^{+} \mathrm{T}$ cells are co stimulated by interaction of $\mathrm{FN}$ with $\mathrm{T}$ cell VIA-4 or VLA-5, and of LN with T cell VIA-6 in a CD3 mAb-mediated proliferation model.

30. DAVIS LS, OPPENHEIMER-MARKS N, BEDNARCZYK $\mathrm{J}$, MCINTYRE

- BW, LIPSKY PE: Fibronectin Promotes Proliferation of Naive and Memory T Cells by Signalling Through Both VLA- 4 and VLA-5 Integrin Molecules. I Immunol 1990. 145:785-793.

FN augments $\mathrm{CD} 3 \mathrm{mAb}$-mediated proliferation of both the naive and memory subsets of T cells by binding to T-cell VLA- 4 and VLA- 5 .

31. Euces MJ, Osborn I, TAKada Y, Crouse C, Luhowsky S, - Hemier ME, LOBB RR: VCAM-1 on Activated Endothelium Interacts with the Leukocyte Integrin VLA-4 at a Site Distinct from the VLA-4/Fibronectin Binding Site. Cell 1990, 60:577-584.

Documents that specific interaction of VLA-4, transfected in K562 cells, with VCAM-1 occurs through a site that is different from that through which the interaction of VLA- 4 with FN occurs.

32. Yamada A, NiKaido T, Nojima Y, Schlossman SF, Morimoto

- C: Activation of Human CD4 T Lymphocytes. Interaction of Fibronectin with VLA-5 Receptor on CD4 Cells Induces the AP-1 Transcription Factor. J Immunol 1991, 146:53-56.

T-cell VLA-5 binding to immobilized FN activates the IL-2 transcription factor AP-1.

33. DUSTIN MI, SPRINGER TA: T-cell Receptor Cross-linking Tran-. siently Stimulates Adhesiveness Through LFA-1. Nature $1989,341: 619-624$.

Reports that TCR-CD3 complex crosslinking increases the avidity of LFA 1 for its ligand, ICAM-1. This increase in avidity is rapid, transient, and not caused by enhanced receptor expression. The induced change of LFA-1 that results in a high avidity binding state is mediated by intracellular signals from the TCR-CD3 complex. This detailed description of an activation-dependent adhesion pathway has major consequences for models of $T$-cell interaction with apposing cells.

34. O'ROURKE AM, ROGERS J, MESCHER MF: Activated CD8 Binding - to Class I Protein Mediated by the T-cell Receptor Results in Signalling. Nature 1990, 346:187-189.

Reports that CD8 binding to class I MHC can provide co-stimulation under conditions of low levels of TCR occupancy.

35. Molngeon PE, Lucich JL, Stebbins CC, ReCNy MA, WallNer

- BP, Koyasu S, ReINHERz EL: Complementary Roles for CD2 and LFA-1 Adhesion Pathways During T Cell Activation. Eur J Immunol 1991, 21:605-610.

In contrast to the rapid increase of adhesion function of LFA-1, the CD2 avidity for LFA-3 is already optimal in resting $T$ cells and is not modified within the first $12 \mathrm{~h}$ following TCR-CD3 complex-mediated activation.

36. DETMERS PA, WRIGHT SD, OISEN E, KIMBALL B, COHN ZA: Aggregation of Complement Receptors on Human Neutrophils in the Absence of Ligand. $J$ Cell Biol 1987, 105:1137-1145.

37. Van KoOYK Y, van de WIel-van Kemenade $\mathbf{P}$, Weder $\mathbf{P}$, KUIJPERS TW, FIGDOR CG: Enhancement of LFA-1-mediated Cell Adhesion by Triggering Through $\mathrm{CD} 2$ or $\mathrm{CD} 3$ on $\mathrm{T}$ Lymphocytes. Nature 1989, 342:811-813. 
T-cell activation through a pair of CD2 mAbs induces a more prolonged induction of high avidity LFA-1 molecules than does crosslinked CD3 mAbs.

38. SHIMtZ Y, VAN SEvENTER GA, HORGAN KJ, SHAw S: Roles of Adhesion Molecules in $T$ Cell Recognition: Fundamental Similarities Between Four Integrins on Resting Human $T$ Cells (LFA-1, VLA-4, VLA-5, VLA-6) in Expression, Binding, and Costimulation. Immunol Rev 1990, 114:109-143.

39. MOURAD W, GeHA RS, ChatLla T: Engagement of Major His- tocompatibility Complex Class II Molecules Induces Sustained, Lymphoctye Function-associated Molecule 1-dependent Cell Adhesion. J Exp Med 1990, 172:1513-1516.

Crosslinking of HIA class II molecules with certain specific mAbs in duces the IFA-1 molecules on monocytes to acquire a high avidity for ICAM-1.

40. SANDERS ME, Makgoba MW, Shaw S: Human Naive and Memory $T$ Cells: Reinterpretation of Helper-inducer and Suppressor-inducer Subsets. Immunol Today 1988, 9:195-199.

41. MORIMOTO C, TORIMOTO Y, IEVINSON G, RUDD CE, SCHRIEBER - M, Dang NH, LETvin NI, SChlossman SF: 1F7, a Novel Cell Surface Molecule, Involved in Helper Function of CD4 Cells. J Immunol 1989, 143:3430-3439.

A new mAb 1F7 (specific for an epitope on CD26) inhibits various $T$. cell dependent activation models in vitro. It recognizes preferentially the memory T-cell subset.

42. Mackay CR, Marston WL, Dudler L: Naive and Memory T - Cells Show Distinct Pathways of Lymphocyte Recirculation. J Exp Med 1990, 171:801 817.

Reports that the expression of cell-surface markers that distinguish human naive and memory T.cell subsets is very similar in sheep. In vivo recirculation experiments indicate that in sheep most cells in the affer ent lymph are of the memory phenotype, whereas a great majority of the cells in the efferent lymph are of the naive phenotype.

43. Bevilaceua MP, Stengelin S, Gimbrone MA JR, Seed B: En- dothelial Leukocyte Adhesion Molecule 1: an Inducible Re. ceptor for Neutrophils Related to Complement Regulatory Proteins and Lectins. Science 1989, 243:1160-1164.

Cloning and characterization of the cytokine-inducible ELAM-1, an LN. adhesion molecule for neutrophil binding to endothelial cells.

44. Shimizu $Y$, Shaw $S$, Graber $N$, gopal TV, horgan KJ - Van Seventer Ga, Newman W: Activation-independent Binding of Human Memory T Cells to ELAM-1. Nature 1991, 349:799-802.

Reports that the memory subset of $\mathrm{CD} 4^{+} \mathrm{T}$ cells exclusively bind to purified ELAM-1. This T-cell binding to ELAM-1 occurs independently of $\mathrm{T}$-cell activation.

45. PICKER LJ, KISHIMOTO TK, SMTH CW, WARNOCK RA, BUTCHER - EC: ELAM-1 is an Adhesion Molecule for Skin-homing $T$ Cells. Nature 1991, 349:796-799.

A unique skin-associated subset of memory $\mathrm{T}$ cells defined by the expression of the cutaneous lymphocyte-associated antigen (HECA 452) binds to ELAM-1 transfectants.

46. DUSTIN ML, SPRUnGer TA: Lymphocyte Function Associated Antigen-1 (LFA-1) Interaction with Intracellular Adhesion Molecule -1 (ICAM-1) is One of at Least Three Mechanisms for T Lymphocyte Adhesion to Cultured Endothelial Cells. $J$ Cell Biol 1988, 107:321-331.

47. OsBorn L, HesSion C, TIZARD R, VASSALLO C, LUHOWSKYJ S, - CHr-Rosso G, LOBB R: Direct Expression Cloning of Vascular Cell Adhesion Molecule 1, a Cytokine-induced Endothelial Protein that Binds to Lymphocytes. Cell 1989, 59:1203-1211. Cloning and characterization of an immunoglobulin-like adhesion molecule VCAM-1, which is induced by IL-1 and TNF- $\alpha$ on human en dothelial cells, and binds lymphocytes.

48. THORNHIIL MH, HASKARD DO: IL -4 Regulates Endothelial Cell

- Activation by $\mathbf{I L}-1$, Tumor Necrosis Factor, or IFN-gamma. $J$ Immunol 1990, 145:865-872.
Reports studies of regulation of the endothelial cell expression of MHC class II, ICAM-1, VCAM-1 and ELAM-1 by IL- 4 alone, or in combination with TNF, IL.1 or INF- $\gamma$.

49. Webb DS, Shimizu Y, van Seventer Ga, Shaw S, Gerrard

-. TL: LFA-3, CD 44, and CD45: Physiologic Triggers of Human Monocyte TNF and IL-1 Release. Science 1990, 249:1295-1297.

Immobilized mAbs against LFA-3, CD44 and CD45 induce resting monocytes to release TNF and IL-1. Documents a direct demonstration of CD2-IFA-3 induced IL-1 secretion by monocytes with plastic-immobilized affinity-purified $\mathrm{CD} 2$. This finding provides evidence that $T$ cell-monocyte interaction is a bilateral activation event.

50. LandIS RC, FRIEDMan ML, FISHER RI, Eluis TM: Induction of Hu- man Monocyte IL-1 mRNA and Secretion During Anti-CD3 Mitogenesis Requires Two Distinct T-cell-derived Signals. $J$ Immunol 1991, 146:128-135.

A study that indicates a two step model of monocyte IL-1 production. The initial signal leads to induction of IL-1 mRNA and is mediated by direct contact of the monocyte with CD3 mAb-activated T-cells, and a subsequent signal is provided by soluble T-cell factors that result in IL-1 secretion.

51. KaWAKam K, Yamamoto Y, KaKimoto K, OnOUE K: Require-. ment for Delivery of Signals by Physical Interaction and Soluble Factors from Accessory Cells in the Induction of Receptor-mediated T-cell Proliferation. Effectiveness of IFN gamma Modulation of Accessory Cells for Physical Interac. tion with T Cells. J Immunol 1989, 142:1818-1825.

Reports three requirements for monocyte-dependent $\mathrm{CD} 3 \mathrm{mAb}$-mediated activation of resting $T$ cells: TCR-CD3 crosslinking; soluble mediators from activated monocytes, such as IL-1 and IL- 6 ; and cell-cell contact with fixed IFN- $\gamma$ activated monocytes. Fixed resting monocytes are incapable of providing this accessory signal. This demonstrates elegantly the importance of cell-cell contact in T-cell co-stimulation by monocytes.

52. Koulova L, CIARK EA, SHU G, DuPONT B: The CD28 Ligand - B7/BB1 Provides Costimulatory Signal for Alloactivation of CD4 + T Cells. J Exp Med 1991, 173:759-762.

This study shows that $\mathrm{CD} 28$ and B7/BB-1 mAbs inhibit allo-activation of $\mathrm{CD} 4^{+} \mathrm{T}$ cells by $\mathrm{B}$ lymphoblasts. It also demonstrates that crosslinking of HLA-DR antigens on resting $B$ cells induces rapid expression of B7/BB-1.

53. Bartlett wC, michael A, mcCann J, yuan D, Claassen E, -. NOELE RJ: Cognate Interactions Between $T$ Cells and B Cells. II. Dissection of Cognate Help by using a Class IIrestricted, Antigen-specific, IL-2-dependent Helper $\mathrm{T}$ Cell Clone. I Immunol 1989, 143:1745-1754

Dissection of murine T-cell help for B cells into two phases, an early cell contact-dependent event that induces B-cell cycle progression into G1 (IL-2 independent) and an IL-2 dependent T-cell production of lymphokines that mediates subsequent $\mathrm{B}$-cell proliferation.

54. NOELIE RJ, MCCANN , Marshall L, BartLeTt WC: Cognate In-. teractions Between Helper T Cells and B Cells. III. Contactdependent, Lymphokine-independent Induction of B Cell Cycle Entry by Activated Helper T Cells. I Immunol 1989, 143:1807-1814.

Cell-cell contact of a CD3 mAb-activated murine Th clone with resting $B$ cells is required to induce mRNA synthesis in the B cell ( $G 0$ to $G 1$ in the cell cycle). Subsequent cytokine production by the activated $\mathrm{Th}$ clone induces progression of the B cell to the S phase. $\mathrm{IL}-4$ is one of the cytokines required for this process.

55. BartleTt WC, MCCANN J, SHEPHERD DM, ROY M, NOELLE RJ: - Cognate Interactions Between Helper T Cells and B Cells. IV. Requirements for the Expression of Effector Phase Activity by Helper T Cells. $J$ Immunol 1990, 145:3956-3962 This study reports that only plasma membranes from activated murine T-cell clones (both Th1-type and Th2-type) provide a cell-cell contactmediated signal that in combination with IL-4, induces B-cell proliferation. This activation-induced, membrane-bound signal is dependent on de novo synthesis of protein. 
56. HOdGKJN PD, YamashtTa LC, COFFMAN RI, KEHRY MR: Separa56. tion of Events Mediating $B$ Cell Proliferation and Ig Production by Using $\mathbf{T}$ Cell Membranes and Lymphokines. $J$ Immund 1990, 145:2025-2034.

Documents that membranes from activated Th cells in combination with $L-4$ and $I L-5$, induce B-cell proliferation and differentiation. An actiation-induced, de novo synthesized, membrane-bound signal on Th cells is involved in cell contact between $T$-cell membranes and $B$ cells

57. KRIEGer Л, GRAmmer SF, GREy HM, ChesnUt RW: Antigen Presentation by Splenic B Cells; Resting B Cells are Ineffective Whereas Activated B Cells are Effective Accessory Cells for T Cell Responses. I Immunol 1985, 135:2937-2945.

58. JENKINS MK, BURRELL E, ASHWELL JD: Antigen Presentation by Resting B Cells: Effectiveness at Inducing $T$ Cell proliferation is Determined by Costimulatory Signals, not $T$ Cell Recéptor Occupancy. I Immunol 1990, 144:1585-1590.

Low density and resting B cells express similar levels of Antigen-MHC class II, but only low density (highly enriched for activated) B cells provide the co-stimulatory signals required for IL-2 production.

59. DAmIE NK, LINSLEY PS, LedBeTter JA: Direct Helper T Cell- induced B-cell Differentiation Involves Interaction Between T-cell Antigen $\mathrm{CD} 28$ and B-cell Activation Antigen B7. Eur $J$ Immunol 1991, in press.

MAb blocking studies demonstrate that the $\mathrm{CD} 28-\mathrm{B} 7$ pathway is involved in T-cell-induced differentiation of allo-antigen-presenting $B$ cells into immunoglobulin-secreting cells.

60. TOHMA S, HIROHATA S, LIPSKY PE: The Role of CD11a/CD18.

- CD54 Interactions in Human $T$ Cell-dependent B Cell ACtivation. I Immunol 1991, 146:492-499.

Documents that LFA-1-ICAM-1 is involved in T-cell-dependent B-cell activation. Using LFA-1 deficient $\mathrm{T}$ cells, the authors establish a role for LFA-1 on the B cell, and ICAM-1 on the T cell.
61. Emile $D$, Wallon $C$ Galanaud $P$, Fischer A OLVE $D$ DELFRAISSY JF: Role of the LFA3-CD2 Interaction in Human Specific B Cell Differentiation. I Immunol 1988, 141:1912-1918.

62. Cerdan C, Martin Y, Braily H, Cotircoli M, Finatetta s - costello R, mawas C, Birg F, olive D; li-ialpha is pro duced by $T$ Lymphocytes Activated via the CD2 Plus a $\overline{\mathrm{I}} \mathrm{F}$ Pathways. I Immunol 1991, 146:560-564.

Late $\mathrm{IL}-1 \propto$ secretion by $\mathrm{T}$ cells is mediated onfy through a combination of CD2 and CD28 mAbs. These mAbs however, are each able, to induce $T$-cell proliferation and TNF- $\boldsymbol{\alpha}$ secretion

63. Eljaafari a, vaquero $C$, telliaud $J$, Bismuth $G$, Hivroz $C$ - Dorval I, BERnard A, Sterkers G: Helper or Cytolytic Func tions can be Selectively Induced in Bifunctional $\mathrm{T}$ Cell Clones. I Exp Med 1990, 172:213-218.

Two distinct pairs of $\mathrm{CD} 2 \mathrm{mAbs}$, which induce different second mes sengers, stimulate CD4 $\mathrm{T}$-cell clones to elicit either helper or cytolytic functions. The helper function is associated with a rise in $\left[\mathrm{Ca}^{2+}\right]_{i}$ and phospholipase $C$ activation, whereas the cytulytic function is incluced in the absence of detectable levels of these second messengers

GA van Seventer and S Shaw, Experimental Immunology Branch, National Cancer Institute, National Institutes of Health, Bethesda, Maryland 20892, USA.

Y Shimizu, Department of Microbiology and Immunology, University of Michigan Medical School, Ann Arbor, Michigan 48109, USA. 